EOMmUn: Communication et organisation



Négociation et médiation dans l'entreprise

\title{
Les médiations
}

Jean Bonafé-Schmitt

\section{OpenEdition}

Journals

Édition électronique

URL : http://journals.openedition.org/communicationorganisation/1914

DOI : 10.4000/communicationorganisation. 1914

ISSN : 1775-3546

\section{Éditeur}

Presses universitaires de Bordeaux

\section{Édition imprimée}

Date de publication : 1 mai 1997

ISSN : 1168-5549

\section{Référence électronique}

Jean Bonafé-Schmitt, « Les médiations », Communication et organisation [En ligne], 11 | 1997, mis en ligne le 26 mars 2012, consulté le 19 avril 2019. URL : http://journals.openedition.org/ communicationorganisation/1914; DOI : 10.4000/communicationorganisation.1914

Ce document a été généré automatiquement le 19 avril 2019

(C) Presses universitaires de Bordeaux 


\title{
Les médiations
}

\author{
Jean Bonafé-Schmitt
}

1 Depuis le milieu des années 1970, on parle de plus en plus de médiation que ce soit dans le domaine de la famille, avec la médiation familiale, dans le domaine judiciaire avec la médiation pénale ou civile, ou encore celui de l'entreprise avec les médiateurs d'entreprise, sans oublier les médiateurs sociaux dans les quartiers (Bonafé-Schmitt 1992). Aucun domaine de la vie sociale ne semble échapper à ce phénomène, comme en témoignent les expériences de médiation dans les établissements scolaires pour prévenir les phénomènes de violence ou encore la mise en place de médiateurs dans les bibliothèques pour favoriser à la fois une meilleure diffusion de la culture et gérer les conflits liés aux fonctionnement de ces institutions (Costil, Garden 1993).

2 Au-delà du phénomène de mode, il convient de s'interroger sur le renouveau de la médiation, car celui-ci s'inscrit dans un contexte de crise des mécanismes de régulation sociale (Bonafé-Schmitt 1992). Il est un fait que les lieux traditionnels de régulation, mais aussi de socialisation comme la famille, l'école, le quartier, la justice sont en crise et nous faisons l'hypothèse que la médiation traduit l'émergence d'un nouveau mode de régulation sociale, un nouveau modèle d'action régissant les rapports entre individus, mais aussi plus généralement les relations entre l'État et la société civile.

3 Ce modèle d'action, que nous avons dénommé "action commune ", (Giraud 1993) ne serait pas fondé sur la rationnalité instrumentale qui a façonné nos sociétés depuis l'avènement de la modernité mais sur une autre forme de rationnalité, de nature communicationnelle, dans le sens ou l'entend Habermas c'est-à-dire fondé sur l'intercompréhension (Habermas 1981). Il ne s'agit pas pour nous de prendre partie dans le débat qui oppose les postmodernistes aux partisans du franchissement d'une nouvelle étape de la modernité, mais de nous interroger à partir du développement de la médiation, sur la recomposition des rapports entre État et société civile (Touraine, 1992).

4 Avant d'aborder ces questions, il convient de cerner le phénomène de la médiation car celui-ci est pluriel : sous ce concept on retrouve à la fois des lieux, des objets, des acteurs, ce qui provoque des confusions conceptuelles entre les activités de médiation liées à la gestion des conflits et plus largement aux problèmes de communication. L'inflation des médiations accentue ce flou conceptuel car il existe une tendance à dénommer médiation 
toute intervention de tiers, ce qui rend nécessaire un effort de clarification des définitions de ce phénomène.

\section{L'inflation des « médiations »}

5 La médiation est à la mode et il ne passé pas un jour sans que l'on voit apparaître un nouveau médiateur mais la multiplication de ce type de fonction ne se fait pas sans problème en raison du flou qui entoure la définition de ce type d'activité.

\section{Un flou conceptuel}

6 Depuis quelques années, l'inflation des activités de médiation a provoqué un flou conceptuel en raison de l'utilisation de cette dénomination pour des fonctions qui vont de la gestion des conflits à celle de communication. On serait tenté de dire que dans les années soixante-dix, ce fut le règne des « animateurs » et depuis les années quatre-vingts, on serait passé à celui des « médiateurs ».

7 Le développement de la médiation ne représente qu'une illustration de la multiplication de l'intervention de ces nouveaux «tiers » dans la gestion des relations sociales que ce soit sous des appellations traditionnelles, comme les négociateurs, conciliateurs, médiateurs mais aussi sous des nouvelles comme les facilitateurs, les personnes-relais, les personnes-ressources... La multiplication de ces nouveaux tiers ne doit pas faire illusion car dans certains cas ces fonctions dissimulent ou traduisent des évolutions ou des revalorisations de fonctions traditionnelles comme les gardiens d'immeuble qui sont devenus des "médiateurs-techniques». À l'image des «techniciens de surface» ces nouveaux gardiens ont pour rôle d'assurer l'entretien des espaces, qui représente l'aspect technique de leur fonction, mais aussi de gérer les conflits de voisinage c'est-à-dire de jouer un rôle de médiateur.

8 Dans le même sens, certains magistrats revendiquent un rôle de médiation, c'est le cas notamment des membres du parquet dans les Maisons de justice qui utilisent les procédures de classement des affaires pour obtenir des auteurs d'infraction qu'ils réparent le préjudice causé à leur victime (Dourlens, Vidal Naquet 1992). D'autres magistrats comme les juges aux affaires familiales, les juges pour enfants, déclarent être des « juges-médiateurs » c'est-à-dire qu'ils tentent de résoudre les conflits qui leurs sont soumis d'une manière consensuelle en recherchant l'adhésion des parties a leur décision. $\mathrm{Au}$ sein de la magistrature, ce phénomène demeure encore marginal mais il traduit toutefois une évolution de la fonction de juger dans le sens d'une recherche d'un mode négocié et non simplement imposé, de règlement des conflits.

9 À côté de ces professionnels traditionnels de la régulation sociale, on a vu émerger au cours de ces dernières années de nouveaux acteurs, comme les médiateurs, les personnesrelais; l'apparition de ces nouveaux tiers dans la gestion des relations sociales traduit la modification de nos systèmes de régulation sociale. En effet, ces nouveaux tiers ont en commun, en premier lieu, d'axer leur intervention sur la communication et non sur les rapports de pouvoir. En second lieu, ils ont aussi pour rôle de créer ou recréer un cadre de communication entre des individus afin d'établir ou de rétablir un lien social. Enfin, ils ont pour objet de créer les conditions pour favoriser l'autonomie des individus et en faire des acteurs de leur propre histoire. 


\section{Les activités de médiation et les instances de médiation}

10 La médiation ne constitue pas encore un mode autonome de régulation sociale, ce qui explique les confusions actuelles entre les personnes qui ont une activité de médiation, comme les juges, les policiers, les gardiens d'immeuble, et celles qui exercent leur fonction au sein d'instances de médiation, comme les médiateurs familiaux, médiateurs pénaux, sociaux.

\section{Les activités de médiation}

11 De nombreux acteurs, professionnels ou non, exercent quotidiennement une activité de médiation dans la mesure où ils interviennent comme des tiers dans la gestion d'un conflit ou pour ré-établir une communication. Nombreux sont les professionnels qui revendiquent un rôle de médiateurs dans leur activité quotidienne qu'ils soient magistrats, policiers ou travailleurs sociaux.

12 L'activité de médiation traduit aussi une évolution des professions dans le sens d'une prise en compte plus grande de l'agir communicationnel et pas simplement de l'action rationnelle instrumentale qui caractérise le fonctionnement des grandes organisations bureaucratiques. C'est le cas notamment des policiers qui sont souvent amenés à jouer un rôle de médiation dans le cadre des « appels au 17 » que ce soit en matière de « différend familial ou de voisinage » pour reprendre la terminologie policière (Bonafé-Schmitt 1992). Pour régler ces conflits, les policiers utilisent les techniques de médiation c'est-à-dire tentent à travers la discussion de trouver une solution qui permet, au moins provisoirement, de calmer les esprits et de rétablir un minimum de paix sociale. La réalisation de ces «médiations rustiques" par les policiers ne permet nullement d'accréditer l'idée que ceux-ci sont des médiateurs car on ne peut pas faire abstraction de leur qualité de gardien de l'ordre public. Ils ont une fonction d'autorité et ils sont conscients, comme les parties en conflit, que s'ils ne parviennent pas à trouver une solution négociée, ils seront amenés à prendre une décision autoritaire.

13 Une analyse similaire pourrait être faite pour les travailleurs sociaux, qui sont amenés de plus en plus à développer des activités de médiation. Ceci est surtout vrai pour ceux qui travaillent dans les quartiers dits " défavorisés » ou en "difficulté » en raison du déficit de communication qui existent entre les populations et les grandes organisations publiques ou privées.

14 Les assistantes sociales sont de plus en plus nombreuses à revendiquer ce rôle de médiation entre les habitants et les administrations publiques ou les organismes privés soit pour faciliter une communication soit pour aider les usagers à faire valoir leurs droits. Comme dans le cas des policiers, les travailleurs sociaux ont une activité de médiation mais ne sont pas une instance de médiation car leur statut, leur mode de fonctionnement, ne leur permettent pas d'être impartiaux, indépendants, comme doivent l'être les médiateurs.

Un éducateur, une assistante sociale, un animateur, en raison de leur statut, sont enserrés dans une hiérarchie, relèvent d'un organisme qui poursuit des objectifs particuliers et leur fonction même, les amène à être partiaux. En effet, un éducateur, par exemple qui 
doit accorder une attention particulière au jeune qu'il encadre ne pourra en aucun cas être un médiateur impartial dans la gestion des relations sociales de l'adolescent.

Il en est de même de ces " personnes relais » qui émergent dans les quartiers à l'initiative d'organismes sociaux ou de collectivités locales et qui le plus souvent, jouent un rôle d'intermédiaire entre les habitants et les administrations (Delcroix, 1995). Elles ont une activité de médiation mais ce ne sont pas des instances de médiation car le flou de leur statut, de leurs modalités d'intervention, mais aussi leur mode de recrutement, ne leur permettent pas d'être des instances de médiation au sens strict du terme.

\section{Les instances de médiation}

Le flou qui entoure le concept de médiation nous a amené à distinguer les «activités de médiation » et les « instances de médiation ». La confusion s'explique essentiellement par le fait que la médiation ne constitue pas aujourd'hui un mode autonome de régulation sociale et représente encore une activité accessoire pour de nombreux professionnels du droit et du social.

Dans le champ de la régulation sociale, la construction de l'identité des médiateurs se fait avec difficulté en raison de l'utilisation instrumentale de cette nouvelle forme d'intervention par bon nombre d'acteurs qui se définissent comme des "médiateurs naturels ». Mais c'est aussi le cas de certains «médiateurs institutionnels », comme ceux mis en place par des sociétés d'assurance et des organismes de transport qui ont un rôle purement instrumental c'est-à-dire la recherche d'une « fidélisation » de la clientèle.

Selon notre conception, le médiateur peut être défini comme un tiers impartial et il se différencie des autres acteurs, dans le sens où son action repose sur un agir communicationnel pour reprendre la terminologie d'Habermas c'est-à-dire en dehors de toute relation de pouvoir (Habermas, 1981). À la différence des autres acteurs de la régulation qui agissent selon une rationnalité instrumentale, les médiateurs fondent leur action sur une rationnalité communicationnelle, sur la recherche d'un consensus, d'une intercompréhension entre les parties (Habermas 1981). Ce type de projet vise, a travers le processus de médiation, à permettre aux personnes de se construire comme acteurs, de promouvoir une capacité d'action, une autonomie dans la gestion de leurs conflits et plus largement de leurs relations sociales.

La construction de l'identité des médiateurs passé aussi par une reconnaissance d'une certaine légitimité de leur action que ce soit de la part des personnes qui ont recours à eux mais aussi de la part des autres acteurs de la régulation. Nous sommes ainsi amené à distinguer trois types de légitimité qui correspondent en fait à trois types de médiation: la légitimité déléguée, la légitimé professionnelle et la légitimité sociale.

21 Dans le cas de la légitimité déléguée, comme son nom l'indique, l'intervention du médiateur est fondée par un texte, ou une autorité judiciaire comme pour la médiation judiciaire civile ou pénale. En ce qui concerne la légitimité professionnelle, l'action du médiateur se fonde sur une compétence professionnelle, comme celle qui est le plus souvent revendiquée par les médiateurs familiaux ou d'entreprises.

22 Enfin dans le cas de la légitimité sociale, l'action des médiateurs est fondée sur la simple reconnaissance par les parties en conflit de la pertinence de ce mode de résolution des conflits, en dehors de toute autre référence extérieure, comme nous pouvons l'observer dans la médiation de quartier ou communautaire. 

par une certaine structuration du champ de la médiation à travers la constitution d'organisations professionnelles, qui agissent comme des quasiordre professionnels en élaborant des codes de déontologie, en organisant des formations et en publiant la liste de leurs membres ${ }^{1}$. C'est surtout dans le domaine de la médiation familiale que la structuration de la médiation s'est faite autour d'une pratique professionnelle afin de sortir la fonction de médiation d'un statut de «semi-professionnel» jouant un "rôle secondaire et dominé » par rapport aux autres acteurs de la régulation sociale » (Dezalay, 1985).

24 Au sein du champ de la médiation, on assiste à des enjeux de pouvoir entre les professionnels du social et du droit pour le contrôle de cette nouvelle profession en essayant de faire prévaloir leur propre compétence technique, leur maitrise d'une forme d'intervention professionnelle. Mais avec cette recherche d'institutionnalisation de la médiation on assiste à des tentatives, comme dans les cas des médiations judiciaires, de faire rentrer dans "l'ordre juridique un domaine de la pratique jusque-là abandonné à des formes pré-juridiques de Solutions des conflits » (Bourdieu, 1986).

\section{Les modèles de médiations}

La médiation est un phénomène pluriel car ce mode de régulation sociale est sous tendue par différentes logiques, différentes rationnalités, ce qui nous a amené à distinguer les formes de médiation liées à la gestion des conflits de celles portant sur la communication.

\section{Les activités de médiation liées à l'activité de gestion des conflits}

C'est dans le champ de la gestion des conflits que l'on mesure le mieux les différentes logiques qui sous-tendent le phénomène de la médiation car on peut distinguer celles relevant d'initiatives publiques et celles relevant d'initiatives privées. Cette opposition ne doit pas être poussée trop loin car dans la réalité la situation est plus complexe et on assiste à un enchevêtrement des logiques publiques et privées. Il n'est pas rare de constater que des projets de médiation mis en œuvre par des acteurs privés soient financés par des organismes publics.

\section{Les logiques étatiques/publiques}

Les instances de médiation mises en œuvre pas les organismes publics sont régies le plus souvent par des logiques instrumentales qu'elles soient de nature gestionnaire ou d'intégration sociale. Sur un plan chronologique, les premiers projets de médiation étaient fondés sur des logiques gestionnaires c'est-à-dire que l'État avec la création de l'institution du Médiateur de la République en 1974 a cherché à répondre à une «mal administration", à améliorer l'efficacité administrative (Bonafé-Schmitt 1992). Cette logique s'est poursuivie au cours des années suivantes avec la création de médiateurs municipaux, comme celui de la Ville de Paris en 1977, le médiateur du cinéma en 1983, le médiateur du livre en 1990 et enfin la dernière en date, les médiateurs scolaires en 1994.

Poursuivant la même logique gestionnaire, l'État, face au contentieux de masse, a créé des instances extrajudiciaires, comme les conciliateurs en 1978 ou les Boites Postales 5000 en 1977 dans le domaine de la consommation (Faget 1992). Par ces initiatives, l'objectif de 
l'État était de dériver la gestion du contentieux de masse de l'institution judiciaire vers d'autres instances. Ce phénomène s'est poursuivi au cours des années quatre-vingts, basé sur une logique de sous-traitance des affaires de la part de l'institution judiciaire en direction d'instances de médiation. C'est le cas notamment dans le domaine pénal avec la sous-traitance d'affaires par les parquets à des associations d'aide aux victimes ou de contrôle judiciaire ou encore dans le domaine civil avec le renvoi d'affaires par des magistrats du siège aux instances de médiation familiale (Faget, 1992).

Il faudra attendre le début des années quatre-vingt-dix, pour assister à l'émergence d'une nouvelle logique de gestion du contentieux à travers la création des Maisons de Justice et du Droit (MJD). Après avoir développé des logiques gestionnaires basées sur une externalisation du traitement $d u$ contentieux, le Ministère de la Justice a voulu promouvoir de nouveaux modes de gestion des conflits au sein de l'institution judiciaire comme les classements sous condition de réparation en matière pénale, les médiations judiciaires...

Mais à travers l'expérimentation des MJD, l'État ne s'est pas seulement contenté d'améliorer l'efficacité de l'institution judiciaire, il a aussi développé une nouvelle politique de gestion des conflits faisant appel à un traitement social et pas simplement judiciaire du contentieux. La création des MJD s'inscrit dans ces politiques de développement social urbain qui visent à recréer du social, de nouvelles solidarités au niveau des quartiers.

Les exemples les plus significatifs de ces nouvelles politiques qui allient des logiques gestionnaires à des logiques d'intégration sociale se retrouvent dans les expériences de médiation pénale et de quartier mises en place d'une manière conjointe par les municipalités et les parquets et qui font appel à des habitants pour remplir la fonction de médiateur. L'archétype de ce projet de médiation nous est donné par l'expérience de Valence en 1985, suivie par celle des Ulis en 1989 qui ont généré un modèle conjoint de gestion des conflits dans les quartiers, associant les municipalités et les parquets pour à la fois lutter contre le sentiment d'insécurité et recréer du tissu social dans les grands ensembles.

\section{Les logiques sociales}

Parallèlement aux initiatives publiques, un certain nombre d'initiatives ont été prises par des acteurs de la société civile que ce soit de la part de professionnels, d'associations ou encore d'entreprises. Ces initiatives sociétales en matière de médiation obéissent aussi à des logiques différentes : des logiques professionnelles, organisationnelles et sociales.

Pour comprendre l'existence de ces pluralités de logiques en matière de médiation, il est nécessaire de ne pas oublier que le champ de la régulation sociale, constitue aussi un marché qui fait l'objet d'enjeux entre les différents acteurs de la régulation sociale que ce soit sur le plan symbolique ou sur le plan financier (Le Roi, 1995). Sur ce marché de la gestion des conflits, les acteurs traditionnels sont directement concurrencés par ces nouveaux venus que sont les médiateurs. Les médiateurs familiaux et les médiateurs en entreprise, à partir de la fin des années 1980, se sont montrés les acteurs les plus actifs pour structurer le champ de la média-bon en fonction d'une compétence professionnelle.

Sur un autre plan, des entreprises privées et publiques, dans le domaine des assurances (UAP, MACIF...) ou des transports (RATP, SNCF...), ont mis en place des médiateurs. La création de telles structures relève d'une logique purement instrumentale car l'objectif 
assigné a ces instances est la gestion des conflits pouvant surgir entre les entreprises et leurs clients. On serait tenté de dire que les "services médiation" ont remplacé les «services consommation » des années soixante-dix dans une perspective de fidélisation des clientèles.

C'est dans le cadre des quartiers, avec la mise en place dès 1986, de «médiateurs sociaux ", à l'initiative d'associations de médiation, comme les Boutiques de Droit à Lyon, que l'on retrouve les projets reposant sur une logique de réappropriation du pouvoir de gestion des conflits par les habitants. Ces expérimentations se distinguent des précédentes en raison de leur volonté d'utiliser la médiation comme processus pour reconstituer de nouveaux lieux de socialisation, de nouvelles solidarités au niveau des quartiers. Ce sont ces mêmes associations qui sont à l'origine des projets de médiation scolaire faisant appel à des élèves et à des membres de la communauté éducative pour gérer les conflits au sein des établissements scolaires (Bonafé-Schmitt 1996).

\section{Les médiations liées à l'activité de communication}

36 Le phénomène de la médiation ne se limite pas simplement à la gestion des conflits, il s'étend aussi à ce que nous avons appelé les activités de communication. La médiation ne peut se réduire à une simple technique de gestion des conflits, c'est aussi un autre mode de communication, de gestion des relations sociales.

\section{Les logiques publiques}

Ce sont surtout les collectivités locales qui ont pris le plus d'initiatives pour développer des processus de médiation visant à améliorer les modes de communication entre les communautés et les organismes publics. Un certain nombre de municipalités ont mis en place des "médiateurs interculturels", qui ont été recrutés parmi les communautés d'origine étrangère, pour améliorer les modes de communication et favoriser une meilleure intégration. Le choix des dénominations n'est pas neutre, car l'appellation "médiateurs interculturels " dissimule en fait l'existence de véritables "médiateurs communautaires». Effectivement, ils sont recrutés le plus souvent dans leurs communautés d'origine et sont amenés à jouer un rôle d'intermédiaire, de passeur, pour favoriser une meilleure intégration de ces populations.

Parmi ces médiateurs visant à favoriser une meilleure communication entre les communautés et les organismes publics, il est souvent fait mention des "personnesrelais » (qui sont le plus souvent des "femmes-relais») qui, dans leur activité sont souvent amenées à jouer un rôle de médiation. Une étude récente a souligné l'ambigüité de la fonction de personnes-relais, en effet le rôle des personnes-relai n'est pas défini clairement, elles ne bénéficient d'aucun statut, cependant, il leur a été proposé de prendre le titre de médiateur et de suivre une formation adéquate (Delcroix, 1995).

A côté des médiateurs interculturels, apparaissent depuis trois ou quatre ans, à l'initiative de l'État ou des municipalités, des «médiateurs culturels» ou «médiateurs en bibliothèque " (Caillet, 1994). La création de ces nouvelles fonctions obéit à une logique instrumentale lorsque cela leur permet par exemple dans le cas des bibliothèques municipales de Lyon, de gérer des "publics difficiles». D'autre part, on peut voir dans l'apparition de cette fonction une évolution du rôle et des missions des bibliothèques (Costil, Garden, 1996). 
40 À Lyon les médiateurs ont été mis en place dans les bibliothèques situées dans les quartiers dits "défavorisés » afin de gérer les conflits avec certains «publics difficiles » pour l'utilisation des lieux et des moyens mis à leur disposition. D'autre part, pour lutter contre le phénomène d'exclusion sociale, il a été demandé à ces médiateurs de promouvoir la lecture en organisant à l'extérieur des bibliothèques des manifestations en direction des adolescents. Ces actions illustrent un changement de politique des bibliothèques qui ne veulent plus limiter leur rôle à une simple gestion d'objets culturels. Elles tentent également de répondre aux besoins des lecteurs et apporter une contribution à la politique de la ville à travers leur spécificité culturelle.

\section{Les logiques sociétales}

41 En matière culturelle, des organismes privés ont engagé un certain nombre d'initiatives pour lutter contre le phénomène d'exclusion sociale. C'est le cas d'ATD-Quart Monde qui a mis en place des «médiateurs du livre » dans les quartiers défavorisés en faisant appel à des habitants pour remplir ces fonctions d'animation culturelle (Kupier, 1996). Ces projets reposent sur une logique de réappropriation des activités culturelles par les personnes exclues et expérimentent un mode d'apprentissage de la lecture basé sur le désir et non sur l'obligation.

Sur un autre plan, celui du domaine du droit, des structures associatives comme les Boutiques de Droit ont mis en place dans les quartiers « défavorisés » des permanences juridiques qui apparaissent comme de véritables « lieux de médiation juridique » (BonaféSchmitt, 1992). À la différence d'autres professionnels du droit qui prennent fait et cause, les juristes des Boutiques de Droit limitent leur rôle à une simple information juridique, en laissant aux usagers le choix de la voie à suivre. Les Boutiques de Droit ont pour objectif, la réappropriation du pouvoir de gérer un conflit. Dans le domaine juridique, l'apprentissage consiste à faire renaître l'autonomie à la personne qui s'estime victime en lui apprenant le maniement des régies juridiques. Ils jouent aussi un rôle de médiation en traduisant les demandes des usagers sur un plan juridique ou en décodant le langage complexe utilisé par les organismes publics ou privés afin de faciliter la communication.

Enfin, il faut mentionner pour mémoire dans le champ de la formation, le courant de la " médiation pédagogique » avec ses diverses variantes qui visent à développer sur le plan pédagogique de nouvelles méthodes d'acquisition des connaissances (Cardinet, 1995).

\section{Les raisons de ce renouveau de la médiation}

La médiation n'est pas un phénomène nouveau, ce mode de régulation sociale a toujours existé, mais ce qui est nouveau aujourd'hui c'est que son développement se déroule dans le contexte particulier de la crise d'un système de régulation sociale basé sur une rationnalisation continue aussi bien des mécanismes de règlement des conflits que des systèmes normatifs.

\section{La crise $d$ 'un système de régulation sociale}

45 À la lecture des statistiques judiciaires ou policières il semblerait que nos sociétés deviennent de plus en plus conflictuelles, mais il faudrait peut-être nuancer ce jugement, car dans le passé bon nombre de conflits étaient régulés au sein de la famille, du quartier, 
de l'école... Les phénomènes d'industrialisation, d'urbanisation, de mobilité sociale, la persistance de la crise, mais aussi l'interventionnisme étatique, ont mis à mal ces lieux traditionnels de socialisation et de régulation.

L'État, dans l'ensemble de ses domaines d'intervention, semble connaître de plus en plus de difficultés pour réguler la vie sociale. Au fil des décennies, l'intrusion de l'État dans tous les pores de la vie sociale, à travers ce que l'on a appelé, la politique de l'ÉtatProvidence, a eu pour conséquence de remettre en cause les formes traditionnelles de sociabilité au profit d'une "sociabilité étatique » et de structurer entièrement la société par et autour de l'État (Ewald, 1986). C'est dans le domaine de la famille que l'on mesure le mieux ce phénomène de désagrégation de ces structures intermédiaires au profit de l'intervention des services de l'État. Par exemple, une des formes de solidarité primaire, comme l'obligation alimentaire entre parents et enfants qui concrétisait une forme de solidarité intrafamiliale est progressivement remplacée par une «solidarité sociale entre générations médiatisée par des instances étatiques" (Commaille, 1982). Dans le même sens, en matière de divorce, l'État pour se substituer au conjoint défaillant, a créé des procédures de recouvrement direct des pensions alimentaires ou l'octroi d'allocations de substitutions qui ont pour effet d'«'assister», de "tutélariser» et de « déresponsabiliser » les débiteurs (Perrin, 1983).

L'interventionnisme étatique a ainsi participé à la dissolution des solidarités primaires et par voie de conséquence à la désagrégation de ces structures intermédiaires. Au cours de ces dernières années, la crise de l'État-Providence a contribué à accentuer ce phénomène de désorganisation sociale. Il en découle que les différentes composantes de la société apparaissent de plus en plus comme figées en monolithes qui ne savent communiquer entre eux que par la médiation de l'État partout sollicitée (Cohen-Tanugi, 1985). Cette situation explique que pour bon nombre de conflits qui étaient autrefois régulés au sein de la famille ou du quartier, les seuls interlocuteurs soient la police, la justice ou encore les travailleurs sociaux.

48 C'est dans ce contexte de crise d'un système de régulation sociale qu'il convient de resituer le développement des différentes formes de médiation à l'initiative, aussi bien de l'État que des acteurs de la société civile. La mise en œuvre du processus de médiation marqué de la part de l'État, une rupture dans ses modes d'intervention, car la médiation repose sur une approche plus consensuelle et moins formaliste de la gestion des relations sociales.

\section{La crise d'un modèle rationnel de régulation et de socialisation}

Le développement de la médiation traduit aussi la remise en cause d'un modèle de régulation sociale, comme en matière de régulation des conflits ou l'État s'est lancé dans une politique de rationnalisation, de judiciarisation des conflits, à travers la création de juridictions spécialisées. Si, a l'origine la création de nouvelles structures visait à accroître l'efficacité de l'appareil judiciaire, à terme le "taylorisme judiciaire», a entrainé une bureaucratisation de la justice. Dans la pratique, ce modèle rationnel, formaliste, hiérarchisé avec tous ses effets induits (longueur des délais, complexité et coût de la procédure) connaît de plus en plus de mal pour prendre en compte et réguler la complexité des rapports sociaux.

C'est surtout dans les quartiers « défavorisés » que l'on mesure le mieux l'effet pervers de cette politique de rationnalisation continue des procédures de régulation des conflits, 
avec une prolifération de structures spécialisées intervenant sans véritable coordination sur un même territoire. Faut-il rappeler que les découpages des arrondissements judiciaires, ne correspondent pas exactement aux circonscriptions sociales ou de la police, et encore moins aux secteurs scolaires. En vertu d'un certain taylorisme social, cette spécialisation à outrance des interventions aboutit dans le meilleur des cas à superposer les actions concernant un même secteur géographique mais sans que les mesures prises fassent l'objet d'une évaluation ou d'un suivi commun.

Chaque institution agit selon ses propres critères de compétence, dans le cadre de son domaine d'action et ce mode de fonctionnement dans la pratique ne permet pas d'appréhender les situations dans leur totalité. Par l'effet du principe de spécialisation, chaque individu est perçu soit comme un usager d'un service particulier, soit comme le bénéficiaire d'un certain type d'intervention ou de prestation.

En matière normative, on assiste aussi à une contestation d'un modèle rationnel de production normative axé sur la production de normes générales et impersonnelles, visant à induire des régies de comportement, de plus, incapables de réguler la complexité croissante des rapports sociaux. Si la juridicisation des relations sociales, à travers l'obtention de droits sociaux, a représenté un progrès par rapport aux solidarités de proximité qui entretenaient des relations de dépendance à l'égard des communautés héritées, comme la famille ou l'ethnie (Laville, 1994). En revanche, une trop grande juridicisation de celles-ci, notamment dans le domaine du « monde vécu » pour reprendre l'expression d'Habermas, aboutit à remplacer la tutelle de l'homme sur la femme et des parents sur celles des enfants par la tutelle des juges (Habermas, 1981).

Ce phénomène de juridicisation des relations sociales s'est accompagné aussi de leur marchandisation c'est-à-dire que les valeurs marchandes ont pénétré tous les rapports sociaux. Ainsi les relations marchandes se sont infiltrées au fil des décennies, dans tous les domaines de la vie sociale, y compris celui du domaine du « monde vécu » comme la famille, l'école. De plus en plus, on constate que les valeurs marchandes se substituent aux valeurs d'entraide familiale, de voisinage, comme en témoignent ces projets visant à développer des emplois de proximité dans le domaine familial ou dans les quartiers.

\section{La médiation : un nouveau modèle de régulation sociale}

54 Le phénomène de la médiation, ne se limite pas simplement à une simple technique de communication ou des gestion des conflits, il est porteur aussi d'un nouveau modèle de régulation d'un nouveau mode d'action annonçant une recomposition des relations entre l'État et la société civile. Nous partageons le point de vue de certains auteurs qui voient dans le développement de ce "nouveau mouvement de la médiation» dans tous les domaines de la vie sociale, un instrument de transformation politique de nos sociétés » (Becker, 1986).

\section{La médiation : un nouveau modèle d'action}

L'analyse du phénomène de médiation ne peut être réduit, ni à une simple technique de gestion des conflits utilisée par les Etats pour étendre leur contrôle social, ni à l'apparition d'un nouvel acteur sur le marché de la gestion des conflits; elle représente 
aussi un nouveau mouvement social, une nouvelle forme d'action commune impliquant une recomposition des rapports entre l'État et la société civile, entre rapports marchands et non marchands, entre sphère publique et sphère privée... La médiation s'inscrit dans ces «activités» et «structures intermédiaires» de gestion des conflits que l'on voit émerger depuis le début des années soixante-dix et qui traduisent une évolution vers un plus grand pluralisme des systèmes de régulation sociale en raison de la complexité de plus en plus grande de nos sociétés.

Cette évolution irait dans le sens d'une différenciation croissante de nos sociétés en soussystèmes sociaux car en devenant moins étatique et plus civile, elles s'engagent vers une plus grande décentralisation et une autonomisation croissante des acteurs sociaux, et des modes de régulation sociale (Touraine, 1996). Dans ce sens, la médiation est porteuse d'une autre conception des acteurs sociaux et des systèmes d'action qui s'apparente à une hybridation entre l'État et la société civile. Ainsi les médiateurs peuvent être définis comme des "acteurs intermédiaires" dans le sens ou leur statut relève aussi bien du public que du privé, qu'ils remettent en cause les notions de professionnalisation et de non professionnalisation, les divisions des disciplines traditionnelles entre professionnels du droit et du social. À la différence de ces derniers, qui utilisent leur expertise comme un mode de domination, les médiateurs s'efforcent de faire des personnes, qui ont recours à eux, des acteurs de leur propre histoire.

La médiation appelle aussi à une recomposition des rapports entre État et société civile à travers la création de « lieux intermédiaires » de régulation sociale qui font appel à la fois à des acteurs publics et privés, à des professionnels et à des bénévoles comme dans le cas de la médiation de quartier ou scolaire (Bonafé-Schmitt, 1996). Ce type de médiation vise à créer des "espaces transversaux » dans lesquels peuvent être mises en œuvre de nouvelles formes de régulation sociale adaptées aux mutations en cours dans nos sociétés (Laville, 1994). En cela les processus de médiation sont porteurs d'une autre conception de l'acteur et de l'action dans le sens où les médiateurs sont des "acteurs de sens", désireux de participer à la reconstitution de nouvelles « structures intermédiaires » entre l'État et les individus (Laville, 1994). Il s'agirait de donner naissance à de nouveaux lieux de socialisation et de régulation, à de nouvelles «communautés existentielles» qui seraient fondées sur des formes de solidarité faisant plus appel à une rationnalité communicationnelle qu'instrumentale (White, 1994).

Nous sommes prudent dans l'utilisation de la notion de communauté, surtout en cette période où les communautés traditionnelles ont été "disloquées perturbées et désorganisées par les conséquences de la rationalité moderne" et où l'on en voit apparaitre de nouvelles, nées du "fondamentalisme religieux, du chauvinisme ethnique, de cultes ou d'autres phénomènes non rationnels » (White, 1994). Toutefois nous faisons l'hypothèse que les processus de médiation seraient à même de produire une nouvelle forme d'action commune, qui permettrait la constitution de nouvelles solidarités, une nouvelle identité commune à travers l'existence de ces « communautés existentielles ».

\section{La médiation : un nouveau modèle de régulation sociale}

La médiation est le plus souvent réduite à une simple technique de gestion des conflits et cette vision restrictive ne permet pas de l'appréhender comme un autre modèle de régulation sociale. L'analyse des processus de médiation montre que celle-ci repose sur un autre paradigme de gestion des relations sociales fondé sur une rationnalité 
communicationnelle et non instrumentale comme dans les modes traditionnels de régulation. sens où il renvoie a "la mise en discussion des actes de langage de manière à rendre possible les compréhension mutuelle entre acteurs" (Deflem, 1994). Sur cette question, les travaux de J. Habermas sur la théorie de l'agir communicationnel nous apportent un éclairage particulier pour analyser le déroulement du processus de médiation. Ce processus repose sur une logique communicationnelle, c'est-à-dire que le rôle du médiateur consiste à créer les conditions processuelles pour permettre une bonne communication orientée vers l'intercompréhension. Dans ce sens, la médiation repose sur l'échange des paroles afin de favoriser une compréhension mutuelle entre les acteurs. "certain nombre d'exigences de validité qui rendent critiquables les actes de parole: un acte de parole bien conforme et compréhensible exige une vérité objective, Une justesse normative ainsi qu'une véridicité et une authenticité subjectives des acteurs communicationnels » (Deflem, 1994). Le processus de médiation ne peut réussir que si les conditions d'une véritable communication sont remplies ce qui implique le choix de moyens visant un consensus c'est a dire des procédures de décision qui tiennent pour majeurs les participants pour mettre en valeur leurs intérêts et pour régler eux-mêmes leurs affaires (Deflem, 1994). Cette élaboration d'une rationnalité fondée sur le consensus ne doit pas être assimilée à la recherche d'un "consensus mou » mais plutôt du «dissensus » afin de parvenir à un accord (Debarbieux, 1995).

Le processus de médiation repose ainsi sur une logique de "déconstructionreconstruction " c'est-à-dire qu'il implique le passage par une phase de "séparation " avant celle de la «reconstruction» de la relation sociale (Duval, 1993). La phase de séparation permet le positionnement de chacun, l'expression du dissensus, car la construction de l'accord implique de faire ressortir aussi bien les points de désaccords, que les points d'accords et il ne s'agit pas de réduire ces désaccords mais de vivre avec eux, de trouver un modus vivendi (Debarbieux, 1995). Au cours de cette phase de séparation, le rôle du médiateur consiste à "créer cette distance, à ouvrir l'espace indispensable aux échanges de toutes natures qui favoriseront l'identification des acteurs, l'analyse des problèmes, le repérage des solutions possibles et le choix de la conduite à tenir entre les parties» (Duval, 1994). Il s'agit pour le médiateur d'ouvrir des espaces de paroles, d'établir un climat de confiance, afin de permettre aux acteurs de s'écouter, de communiquer, de faire connaître leurs points de vue.

Ce n'est qu'à la suite de la mise en œuvre de cette première phase de séparation que pourra intervenir la phase de reconstruction de l'accord, la recherche d'une solution consensuelle basée sur l'intercompréhension. C'est seulement à travers la constitution de ces espaces de paroles que constituent les rencontres directes entre les acteurs que pourra se construire l'échange réparateur, et d'une certaine manière la reconstitution du lien social (Vaillant, 1994). Au cours de cette phase le médiateur joue un rôle actif en utilisant toutes les techniques communicationnelles, allant de la distribution de la parole, à la traduction et à la reformulation des demandes, sans oublier la gestion des émotions. Cela implique de la part du médiateur à la fois qu'il fasse preuve de distanciation mais aussi de décentration par rapport à ses propres représentations et valeurs et celles des acteurs en présence dans le processus de médiation. En ce sens, la réussite du processus de médiation dépend en grande partie de la posture du médiateur, qui doit faire preuve 
d'une certaine impartialité, favoriser l'instauration d'un climat de confiance, aider à la mise en oeuvre de processus coopératif pour la recherche de solutions consensuelles aux problèmes posés.

64 La médiation s'apparente ainsi, à un véritable rituel, fait d'interactions, d'échanges de paroles que doit maîtriser le médiateur notamment dans les cas oü les acteurs utilisent d'une manière instrumentale le processus pour parvenir a leurs fins. Ce type de situation, illustre d'une certaine manière le rôle central joué par le médiateur, et surtout la nécessaire définition d'une éthique afin de préserver la logique communicationnelle de l'activité de médiation orientée vers l'intercompréhension.

\section{La médiation une nouvelle ideologie?}

65 Nous l'avons $\mathrm{vu}$, toutes les formes de médiation ne reposent pas sur cette logique communicationnelle, et certaines d'entre elle sont fondées sur des logiques instrumentales ce qui explique les critiques de certains adversaires du processus de médiation. En matière de régulation sociale, la médiation demeure un phénomène mineur, mais cela n'empêche pas qu'elle représente un enjeu de pouvoir pour de nombreux acteurs sociaux.

Pour l'État, les différentes formes de médiation judiciaire permettent à celui-ci de gérer d'une manière plus consensuelle les conflits, mais aussi de développer de nouvelles formes d'interventions dans les relations sociales, de nouvelles formes de contrôle social. La médiation participe ainsi au renouvellement des formes de pacification des relations sociales de la part de l'État, en faisant appel à ces nouveaux acteurs que sont les médiateurs. C'est pour cette raison que les Etats se sont lancé dans une politique d'institutionnalisation et de financement des structures de médiation, comme en témoigne le vote en France, de la loi de 1993 pour la médiation pénale et celui de 1995 en matière civile. Toutefois, la promulgation tardive de ces textes, une dizaine d'années après l'apparition des premières expériences, traduit les résistances que suscite ce nouveau mode de régulation aussi bien à l'intérieur qu'à l'extérieur de l'État.

D'autres acteurs sociaux, comme les groupes religieux, ont utilisé la médiation comme médium pour promouvoir un nouveau modèle de justice: la justice restauratrice ou réparative (restorative justice). C'est dans les pays anglo-saxons, comme les États-Unis ou la Grande-Bretagne que le phénomène est le plus visible avec le rôle joué par les Ménnonites et les Quakers dans le développement du mouvement "Victim-Offender Médiation ». A l'origine, le mouvement se dénommait "Victim-Offender Reconciliation Program » (VORP) et comme son nom l'indique il était fondé sur deux principes de base : la réconciliation et la communauté (Umbreit, Coates, 1992).

Avec ces programmes de médiation/réconciliation, il s'agirait de rompre avec le modèle de justice criminelle privilégiant principalement les intérêts de l'État, pour revenir à une conception de la justice faisant une plus large place aux parties en conflit et à la communauté. Selon eux, la majorité des infractions sont commises par des personnes vivant au sein de la même communauté. Par conséquent, ces problèmes doivent être réglés par les membres de cette communauté et non par des professionnels qui en sont extérieurs (Bonafé-Schmitt, 1995). En France, le phénomène religieux est moins visible si ce n'est que la seule confédération syndicale ouvrière qui a organisé un colloque sur la médiation, est la Confédération Française des Travailleurs Chrétiens (CFTC). 
69 Au sein de la société civile, un autre groupe social s'est fait l'avocat de la médiation, il s'agit des organisations de pères divorcés. Ces dernières considèrent qu'en matière d'affaires familiales, l'institution judiciaire en raison de son mode de fonctionnement et de ses valeurs, est mal adapté pour gérer ce type de contentieux et a tendance à privilégier les intérêts de la femme au détriment de l'homme. Sans prétendre que ces associations de parents divorcés utilisent d'une matière instrumentale la médiation pour défendre leurs intérêts spécifiques, elles considèrent que le contentieux lié à la famille, en raison de sa nature, devrait relever de modes consensuels de règlement des conflits.

De leur côté, les associations féministes, surtout aux États-Unis, sont très critiques à l'égard de la médiation car elles considèrent que l'institution judiciaire offre des garanties procédurales permettant de mieux protéger les intérêts de la femme (Fineman, 1988). Elles considèrent que les processus de médiation ne font que reproduire les inégalités de pouvoir existant entre l'homme et la femme sur le plan social ou économique.

71 Il existe aussi bien en France qu'aux États-Unis un fort courant critique de la médiation qui considère que celle-ci sécrète une idéologie de l'harmonie qui ne prend pas en compte l'inégalité des pouvoirs dans la société (Abel, 1981). Cette idéologie de l'harmonie repose sur la négation des conflits car son objet n'est pas de prévenir les causes de ceux-ci mais leur expression. Est ainsi dénoncée une vision de la justice qui encourage les parties à ne voir dans les procédures judiciaires, que de l'aliénation, de l'hostilité et des coûts excessifs, et dans la médiation un processus qui encourage les responsabilités civiques et communautaires. Cette vision idéologique de la médiation transforme les conflits en des problèmes de communication plutôt que des luttes sur des valeurs, des intérêts. En un mot, les conflits sur les faits et les droits se transforment en différends relationnels ou affectifs. L'idéologie de l'harmonie serait porteuse d'un certain modèle de société basée sur la croyance que chacun partagerait les mêmes objectifs, valeurs, et qu'ainsi serait favorisée une meilleure pacification des populations.

\section{Conclusion}

72 Pour analyser le développement de la médiation, on ne doit pas en avoir une vision trop fonctionnaliste car cela ne nous permettrait pas de comprendre que celle-ci est un phénomène pluriel et qu'elle s'inscrit dans une crise de notre système de régulation sociale. La médiation ne vise pas à répondre à des dysfonctionnements de ce système, mais à proposer un autre modèle de régulation sociale basé sur une rationalité de nature communicationnelle. Elle traduit aussi l'émergence d'un nouveau mode d'action régissant les rapports entre l'État et la société civile, basés sur la décentralisation, la déprofessionnalisation, la déjuridicisation. Cette conception de la médiation repose sur l'hypothèse que la diversité et la complexité de la vie sociale encouragent le développement de modes décentralisés de régulation des relations sociales permettant ainsi aux individus de se réapproprier les modes de gestion des conflits.

73 Ce modèle de médiation devrait permettre la reconstitution de structures intermédiaires entre l'État et les individus et la création de nouvelles solidarités. La médiation remet aussi en cause une forme de professionnalisme, qui sous le couvert de l'expertise vise à déposséder les individus de la gestion de leur conflit, et permet au contraire de reconstruire leurs capacités d'action individuelle et d'être des acteurs a part entière de leur conflictualité. Enfin, la médiation est porteuse d'un nouveau système normatif qui 
fait plus appel au notions d'équité qu'à celles tirées du droit positif. En effet, la reconnaissance des structures de médiation comme «fait normatif, implique que l'on admette que les accords puissent régler les rapports entre les parties d'une manière différente que le droit positif en faisant appel aux règles d'équité, aux usages et pratiques locales (Gurvitch, 1988).

Les processus de médiation paraissent plus adaptés à la résolution des nouvelles formes de conflictualité qui traversent nos sociétés (Touraine, 1992). Il apparaît que les conflits qui touchent les sphères de la reproduction matérielle comme les conflits du travail, ont perdu de leur centralité dans nos sociétés, au profit de nouveaux conflits qui surgissent dans "les sphères de la reproduction culturelle, de l'intégration sociale et de la socialisation" comme en témoignent les quasi-émeutes urbaines de Vaulx en Velin en France ou de Los Angeles aux États-Unis (Habermas, 1981).

Les institutions traditionnelles de régulation sociale rencontrent de plus en plus de difficultés pour réguler ces nouveaux types de conflits car ceux-ci ne naissent pas «de problèmes de redistribution, mais de questions qui touchent à la grammaire des formes de vie»" (Habermas, 1981). Ils posent de nouveaux problèmes liés aux formes d'exclusion sociale, de phénomène de précarisation, de fragmentation des identités, mais aussi liés à la qualité de la vie, l'égalité de droits, la réalisation individuelle de soi, l'identité sociale (Habermas, 1981). Pour faire face à ces nouveaux problèmes, il est nécessaire de créer de nouveaux lieux, de nouvelles structures de régulation, de nouvelles formes de médiation fondées sur une rationnalité non plus instrumentale mais communicationnelle.

\section{BIBLIOGRAPHIE}

ABEL R., Conservative conflict and the reproduction of capitalism : the role of informal justice, UCLA Law Review sept. 1981.

BABU A, BASTARD B., (sous la direction de), La médiation dans tous ses états, Le Groupe Familial n - 125/1989,138 p.

BASTARD B., Cardia-Voneche (L.), Le divorce autrement : la médiation familiale, Syros-alternatives, 1990.

BONAFE-SCHMITT Jean-Pierre, La médiation : une justice douce, Syros-alternatives, 1992.

BONAFE-SCHMITT Jean-Pierre, « La gestion policière de l'insécurité » in Insécurité et habitat, sous la direction de Y. Bernard, Editions de l'Espace Européen, Paris, 1992.

BONAFE-SCHMITT Jean-Pierre, Les Boutiques de Droit, Informations Sodales n²2/1992.

BONAFE-SCHMITT Jean-Pierre, Le mouvement du Victim-Offender Médiation ; l'exemple du Minnesota Citizen Council on Crime and Justice, Droit et Société, n 9/1195.

BONAFE-SCHMITT Jean-Pierre., La médiation scolaire : un processus éducatif Revue de Psychologie de la Motivation, premier semestre 1996. 
BOURDIEU Pierre, La force du droit. Eléments pour une sociologie du champ juridique, Actes de la Recherche en Sciences Sociales $n^{\circ} 64: 1986$.

CAILLET Elisabeth, Les médiateurs culturels dans les musées, Bulletin Bibliothèque France t. 39, n • 5/1994. ; « Costil F, Garden A) » Une aventure et une réussite menées à Lyon ».

CARDINET A., Pratiquer la médiation en pédagogie, Dunod, Paris, 1995,186 p.

COHEN-TANUGI, Le droit sans l'État, PUF, Paris, 1985.

COMAILLE J., Permanence et redéfinitions du droit et de la justice face a la famille, Actes, n 35-36/1982.

COSTIL F., Garden A., Une aventure et une réussite menées à Lyon, Bulletin d'Informations de l'Association des Bibliothécaires de France, n 170/1996.

DEBARBIEUX E., Pratique de recherche sur la violence à l'école par une médiation sociologique, Skholé, $\mathrm{n}$ - 2/1995.

DEFLEM M., La notion de droit dans la théorie de l'agir communicationnel de jürgen Habermas, Déviance et Société, 1994, vol. 18, nº 1 .

DELCROIX C., et alii, Synthèse de la recherche nationale portant sur : Rôles et perspectives des femmes-relais en France, ADRI, 1995.

DEZALAY Yves, Vers une sociologie de la production de droit par et Pour les professionnnels de la médiation juridique, Annales de Vaucresson n²3/1985/2.

DOURLENS C, Vidal-Naquet P, L'autorité comme prestation. La justice et la police dans la politique de la ville, CERPE, 1993,189 p.

DUVAL E., « L’espace intermédiaire » in La Médiation, Non Violence Actualités, Montargis, 1993.

EWALD Francois, L'Etat-Providence, Grasset, Paris, 1986.

FAGET Jacques, Justice et travail social. Le rhizome pénal, Erés, Toulouse, 1992.

FINEMAN M., Dominant discourse, professional language, and legal change in child custody decision making, Harvard Law Review, vol. 101, n 4/1988.

GIRAUD Claude, L'action commune, L'Harmattan, Paris, 1993.

GOLBERG S., GREEN E., SANDER E, Dispute Resolution, Little Brown and Company, Boston, 1985.

GURVITCH Georges, «L'idée de droit social » in qui a peur de l'autogestion, 10/18.

GUILLAUME-HOFNUNG M., La médiation, Que sais-je ?, PUF, 1995,127 p.

HABERMAS Jurgen, Théorie de l'agir communicationnel, Tome 2 Pour une critique de la raison fonctionnaliste, Fayard, Paris, 1981 p. 393.

HAMMOUCHE Abdelhafid, La politique de la ville entre médiations et proximités, article à paraître.

UMBREIT M., COATES R., Victim-Offender Mediation : an analysis of programs in four states of the US, Citizen Council Mediation Services, School of Social Work, University of Minnesota, 1992.

KUPIER A., Les médiateurs du livre, analyse des activités, Bulletin d'Informations de l'Association des Bibliothécaires de France » n 170/1996 p. 70.

LAVILLE J-L., Economie solidaire et crise de l'État en Europe, Lien Social et Politiques-RIAC 32/1994.

LE ROI E., La médiation, mode d'emploi, Droit et Société n² 29/1995 
Messmer H and Otto H-U. (editors), Restorative Justice on Trial-pitfalls and potentials of victim-offender mediation-International research perspectives, edited by, Kluwer Academic Publishers, Dordrecht, 1992, Netherlands, $590 \mathrm{p}$.

MOAL A., L'éducabilité cognitive, de la méthode à la démarche, Bulletin régional de l'association nationale des communautés éducatives, $\mathrm{n}^{\circ}$ spécial/1994.

PERRIN J-F., « Tendance des changements législatifs en matière de divorce en Europe occidentale » in COMAILLE J et alii Le divorce en Europe occidentale. La loi et le nombre, INED, Paris, 1983.

PERRIN J-R, WIDMER P, (sous la direction de), La médiation : un mode alternatif de résolution des conflits, Publications de l'Institut suisse de droit comparé, 19, Genève, 1992.

SIX Jean-Francois, Le temps des médiateurs, Seuil, 1990.

SIX Jean-Francois, Dynamique de la médiation, Desclée de Brouwer-Culture de Paix, Paris, 1995, $281 \mathrm{p}$.

TOPOR Lucienne, La médiation familiale, PUF, Que Sais-Je ?, nº 2663,1992, 128 p.

TOURRAINE A., Critique de la modernité, Fayard, Paris, 1992.

TOURRAINE A., Le nationalisme contre la nation, L’Année Sociologique 1996, nº 1.

TOUZARD Hubert, La médiation et la résolution des conflits, PUF, Paris, 1977, 420 p.

VAILLANT M., sous la direction de « De la dette au don. La réparation pénale à l'égard des mineurs » ESF éditeur, Paris, 1994.

WRIGHT M., and Galaway B. (editors), Mediation and criminal justice, Sage publications, 1988, 304 p.

Médiation interculturelle : actes de la formation 1992-93, EN FAS Revue de l'Education NationaleFonds d'Action Sociale, $\mathrm{n}^{\circ}$ 5, $130 \mathrm{p}$.

\section{NOTES}

1. Les principales organisations de médiateurs sont: l'Association pour la Promotion de la Médiation Familiale (APMF) et le Comité National des Services de Médiation Familiale (CNSMF) en matière de médiation familiale. L'Institut National d'Aide aux Victimes et de Médiation (INAVEM) et le Comité de Liaison des Associations de Contrôle Judiciaire (CLCJ) en matière de médiation pénale. LeCentre National de la Médiation, qui a axé surtout son action sur la formation des médiateurs et la publication d'un code de déontologie et il regroupe des associations et groupements de média -bon Le Réseau des Médiateurs Associés (RMA) qui regroupe des médiateurs familiaux, de quartier, d'entreprise... Le Réseau International des Médiateurs d'Entreprise (RIME) qui a axé son action en direction des entreprises. L'Association pour l'Echange et la Médiation Interculturelle qui a axé son action en direction des médiateurs interculturels L'Association des Conciliateurs de France qui tente de regrouper les conciliateurs. 


\section{RÉSUMÉS}

Depuis le milieu des années 1970, on parle de plus en plus de médiation. Sous cette dénomination on retrouve à la fois des lieux, des objets, des acteurs, ce qui provoque des confusions conceptuelles. Au-delà du phénomène de mode, il convient de s'interroger sur le renouveau de la médiation, car celui-ci s'inscrit dans un contexte de crise des mécanismes de régulation sociale. Il est un fait que les lieux traditionnels de régulation, mais aussi de socialisation, comme la famille, l'école, le quartier, la justice... sont en crise et nous faisons l'hypothèse que la médiation traduit l'émergence d'un nouveau mode de régulation sociale, un nouveau modèle d'action régissant les rapports entre les individus, mais aussi plus généralement les relations entre l'État et la société civile.

Since the mid of the seventies, we are talking more and more about mediation. Through that calling, we gather places, things, actors which are arousing conceptual disorders. Beyond a trendy phenomenon we question about mediation revival because of its place into crise's context of social regulation's mecanism. It is a fact that traditional places and also socialization as family, school, district, justice... are in crise and we are going on the assumption that mediator emphasizes a new way of social regulation, a new action pattern governing human relationship, but more gene-rally relationship between State and civil Society.

\section{AUTEUR}

\section{JEAN BONAFÉ-SCHMITT}

Jean Bonafé-Schmitt est chercheur au GLYSI-Sociologies et Anthropologies des formes d'Actions, une équipe du CNRS associée a l'Université Lyon II. Spécialisé dans la sociologie juridique, il a particulièrement axé ses recherches dans le domaine de la résolution des conflits et de la production des normes. Au cours de ces dernières années, il a surtout étudié le phénomène de la médiation à partir d'une approche comparative entre la France et les États-Unis. 